\title{
Digital signal processing approaches for semiconductor phase noise tolerant coherent
} transmission systems

\author{
Iglesias Olmedo, Miguel; Pang, Xiaodan; Schatz, Richard; Zibar, Darko; Tafur Monroy, Idelfonso ; \\ Jacobsen, Gunnar; Popov, Sergei
}

\section{Published in:}

Proceedings of the SPIE

Link to article, DOI:

$10.1117 / 12.2078408$

Publication date:

2015

Document Version

Publisher's PDF, also known as Version of record

Link back to DTU Orbit

Citation (APA):

Iglesias Olmedo, M., Pang, X., Schatz, R., Zibar, D., Tafur Monroy, I., Jacobsen, G., \& Popov, S. (2015). Digital signal processing approaches for semiconductor phase noise tolerant coherent transmission systems. In Proceedings of the SPIE (Vol. 9388). [93880A] SPIE - International Society for Optical Engineering. Proceedings of SPIE - The International Society for Optical Engineering https://doi.org/10.1117/12.2078408

\section{General rights}

Copyright and moral rights for the publications made accessible in the public portal are retained by the authors and/or other copyright owners and it is a condition of accessing publications that users recognise and abide by the legal requirements associated with these rights.

- Users may download and print one copy of any publication from the public portal for the purpose of private study or research.

- You may not further distribute the material or use it for any profit-making activity or commercial gain

- You may freely distribute the URL identifying the publication in the public portal 


\title{
Digital signal processing approaches for semiconductor phase noise tolerant coherent transmission systems
}

\author{
Miguel Iglesias Olmedo(1,2), Xiaodan Pang( ${ }^{(3)}$, Richard Schatz ${ }^{(1)}$, Darko Zibar ${ }^{(2)}$, Idelfonso \\ Tafur Monroy ${ }^{(2)}$, Gunnar Jacobsen ${ }^{(1,3)}$, Sergei Popov ${ }^{(1)}$ \\ (1) Optics division, Royal Institute of Technology (KTH), Electrum 229, Kista, SE-164 40, \\ Sweden. \\ (2) DTU Fotonik, Technical University of Denmark (DTU), 2800 Kgs. Lyngby, Denmark. \\ (3) Networking and Transmission Laboratory, Acreo AB, SE- 16425 Kista, Sweden.
}

\begin{abstract}
We discuss about digital signal processing approaches that can enable coherent links based on semiconductor lasers. A state-of-the art analysis on different carrier-phase recovery (CPR) techniques is presented. We show that these techniques are based on the assumption of lorentzian linewidth, which does not hold for monolithically integrated semiconductor lasers. We investigate the impact of such lineshape on both 3 and $20 \mathrm{~dB}$ linewidth and experimentally conduct a systematic study for 56-GBaud DP-QPSK and 28-GBaud DP-16QAM systems using a decision directed phase look loop algorithm. We show how carrier induced frequency noise has no impact on linewidth but a significant impact on system performance; which rises the question on whether 3-dB linewidth should be used as performance estimator for semiconductor lasers.
\end{abstract}

Keywords: optical communications, coherent communications, phase noise

\section{INTRODUCTION}

Cloud services have already revolutionized the way modern societies interact, yet this is just the beginning of a much bigger data communication revolution. Smart-grid, smart-city and autonomous transportation are feasible near-term solutions to reduce global energy consumption and therefore our environmental footprint. These approaches rely on enormous amounts of data being sensed and transported throughout the city, alongside next generation broadband services, compromising the ability of current metropolitan area networks to cope up with bandwidth demands. Pushing coherent technologies towards the end-user could effectively tackle the problem, but the strong requirements on laser linewidth make them rely on costly external cavity lasers (ECLs), rendering the transceiver economically nonviable for metro-access scenarios. Monolithically integrated semiconductor lasers on the other hand, are generally more cost-effective, energy efficient, and easy to integrate, at the expense of higher linewidths due to carrier induced frequency noise. Consequently, realizing cost-competitive coherent data-links on the range of 100-500 km based on semiconductor lasers has become a timely research question.

Digital signal processing (DSP) is one of the key technologies that enabled the commercialization of coherent technologies on the past decade. ${ }^{1}$ A critical block in the DSP chain is the CPR. It is normally the last block before symbol decision takes place, and it is in charge of compensating for the laser phase noise, which is directly related to the frequency noise. Typically, those algorithms are evaluated in terms of optical signal-to-noise ratio (OSNR) penalty versus the laser linewidth times the symbol period of the transmitted signal $\left(\Delta_{v} \tau\right)$. The laser linewidth is the full width half maximum (FWHM) of its optical spectrum. It is an important figure of merit for lasers generally used to quantify the amount of frequency noise that is present in a coherent transmission link, which plays an important role on system performance. ${ }^{2}$ Under the assumption of spectrally flat white frequency noise, the laser linewidth is directly proportional to the power of the frequency noise. ${ }^{3}$ This assumption holds relatively well for ECLs, which are often used in coherent transceivers as they can provide very narrow linewidths. ${ }^{4}$ For that reason, it makes sense to use $\left(\Delta_{v} \tau\right)$ to benchmark CPR algorithms. On the other hand, when considering monolithically integrated semiconductor lasers, the power spectral density of the frequency noise is no longer

Further author information: (Send correspondence to Miguel Iglesias Olmedo.)

Miguel Iglesias Olmedo.: E-mail: miguelio@kth.se, Telephone: +34635164651

Optical Metro Networks and Short-Haul Systems VII, edited by Atul K. Srivastava, Benjamin B. Dingel, Achyut K. Dutta, Proc. of SPIE Vol. 9388, 93880A · C 2015 SPIE · CCC code: 0277-786X/15/\$18 · doi: 10.1117/12.2078408 
flat, and hence the relationship between linewidth and frequency noise is no longer trivial. ${ }^{5,6}$ Hence, it is an open question whether linewidth is a good estimator of performance in this case.

In this paper, we review the state of the art in CPR techniques and investigate the relation between frequency noise power spectral density, laser linewidth, and system performance when semiconductor lasers are considered. We first derive a frequency noise model that takes into account carrier induced frequency noise and flicker noise. Then, we numerically calculate the laser lineshape while varying different parameters of the model, and analyze the impact on 3-dB and 20-dB linewidths. Finally, we experimentally investigate the impact of different frequency noise profiles on system performance for 28 and 56 Gbaud QPSK systems.

\section{CARRIER PHASE RECOVERY TECHNIQUES}

The most optimum phase estimator that we can use is the maximum a posteriori (MAP). ${ }^{7}$ This involves estimating both the data (symbols) and the phase, and it is not feasible for real-time DSP implementation in a coherent receiver. Nevertheless this method provides an optimum performance as a reference for other methods. Practical implementations of CPR algorithms estimate the phase separately from the data. That means that the data must be removed from the phase noise sequence before the estimation process begins. This can be achieved by using either feedback or feedforward loops. A commonly used algorithm that belongs to the feedback family is the decision directed phase lock loop (DDPLL), ${ }^{8-11}$ where the phase estimator uses the estimated carrier phase of a previous symbol to derotate the current symbol, feeding the result into a decision block. This method has the advantage of being able to track the phase, allowing for moderate frequency offset compensation and reducing the probability of cycle slips. However, for this to be effective, the feedback delay needs to be very low, which imposes several challenges for real time implementation. Decision aided maximum likelihood $(\mathrm{DA} M L)^{12,13}$ is another feedback based algorithm that is more tolerant to non-linear phase noise. Although computationally less demanding, its performance is still tightly related to the feedback delay. Recently, Kalman filtering has been proposed for CPR. ${ }^{14}$ It can be seen as a generalized DDPLL where the loop gain is variable and both the phase and the amplitude are estimated. This method was compared to feedforward structures and was shown to outperform them at low OSNR values. A more practical approach to CPR for implementation purposes is the feedforwrad structure, since it avoids the need for feedback delays. ${ }^{15}$ The most popular technique is the so-called Viterbi \& Viterbi algorithm, ${ }^{16}$ which works best for M-phase-shift keying (PSK) signals. The algorithm removes the data information by squaring the received sequence to the $\mathrm{M}^{\text {th }}$ power, and estimates the phase by calculating the average phase of the resulting cluster in the constellation and dividing it by M. The simplicity of this method is overshadowed by its computational complexity due to the non-linear operations, and its inapplicability to quadrature amplitude modulation (QAM) signals. This last disadvantage was quickly overcome with the two-stage quadrature phase-shift keying (QPSK) partitioning scheme. ${ }^{17}$ The use of two-stage feedforward techniques is now gaining popularity. The first stage is aimed to erase the modulation data whereas the second stage is used to estimate the phase. ${ }^{18-20}$ Blind phase search (BPS) is a simple method used in the second stage. It is a brute force approach in which a finite number of test phases are applied to the constellation until minimum error vector magnitude (EVM) is reached.

All of these techniques, however, assume lorentzian linewidth; and their performance may be degraded in presence of high carrier induced frequency noise. It is therefore necessary to study the influence of semiconductor frequency noise on both laser linewidth and system performance.

\section{SEMICONDUCTOR LASER FREQUENCY NOISE MODEL}

The short-term phase variation of a waveform, can be represented either as phase or frequency noise. Frequency noise refers to random fluctuations of the instantaneous frequency, which is the temporal derivative of the phase. We model the single-sided power spectral density of frequency noise as:

$$
S_{v}(f)=\frac{10^{9} \Delta_{v(1 / f)}}{\pi f}+\frac{\Delta_{v i n t}}{\pi\left(1+\alpha^{2}\right)}\left(1+\alpha^{2} \frac{f_{R}^{4}}{\left(f_{R}^{2}-f^{2}\right)^{2}+\left(\frac{K f_{R}^{2}}{2 \pi} f\right)^{2}}\right)
$$

where: 

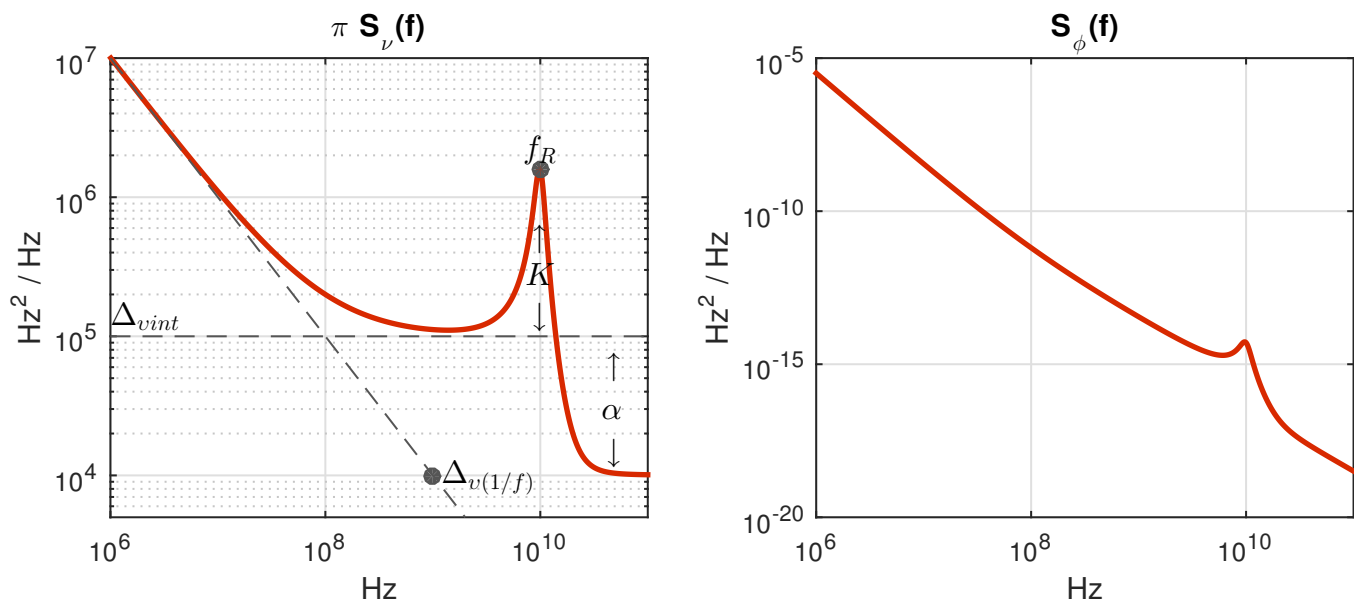

Figure 1: (left) Frequency noise power spectral density (PSD) for $\Delta_{v(1 / f)}=10 \mathrm{kHz}, \Delta_{v i n t}=100 \mathrm{kHz}, f_{R}=10$ $\mathrm{GHz}, K=0.15$ ns and $\alpha=3$. (right) Phase noise PSD obtained by dividing $S_{\nu}(f) / f^{2}$

- $\Delta_{v(1 / f)}$ describes the level of $1 / f$ noise at $1 \mathrm{GHz}$.

- $\Delta_{\text {vint }}$ describes the level of the intrinsic frequency noise at low frequencies.

- $f_{R}$ is the resonance frequency.

- $K$-factor describes how the damping rate increases with relaxation frequency. The $K$-factor of semiconductor lasers is approximately bias independent and in the range of 0.1-1 ns.

- $\alpha$ parameter determines the level of white frequency noise beyond the resonance frequency.

Figure 1 shows the frequency and phase power spectral density (PSD) for a frequency modulation (FM) noise model with parameters $\Delta_{v(1 / f)}=10 \mathrm{kHz}, \Delta_{\text {vint }}=100 \mathrm{kHz}, f_{R}=10 \mathrm{GHz}, K=0.15 \mathrm{~ns}$ and $\alpha=3$. It is a common practice to scale the result by $\pi$ in order to match the intrinsic linewidth $\left(\Delta_{\text {vint }}\right)$ with what would be the 3-dB linewidth if the lineshape was Lorentzial. The phase noise PSD is obtained by simply dividing the frequency noise PSD by $f^{2}$.

\section{IMPACT ON LINEWIDTH}

If the frequency noise PSD is known, the optical spectrum can be calculated as the Fourier transform of the autocorrelation function: ${ }^{6}$

$$
S_{v}(\Delta v)=\text { Fourier }[R(\tau)] \propto \text { Fourier }\left\{\exp \left[-2(\pi \tau)^{2} \int_{0}^{\infty} S_{v}(f)\left|\frac{\sin (\pi f \tau)}{\pi f \tau}\right|^{2} d f\right]\right\}
$$

where $S_{v}(\Delta v)$ is the optical spectral density, $\Delta v=v-v_{0}$ is the optical frequency measured with laser frequency $v_{0}$ as a reference, and $S_{v}(f)$ is the frequency noise PSD as described in section 3.

Figure 2 shows the calculated optical lineshape for different frequency noise PSDs. For the blue curve, only spectrally flat white frequency noise is considered. This yields to a Lorentzial lineshape with a 3-dB linewidth of $\pi h_{0}$, where $h_{0}$ is set to $100 \mathrm{kHz}$. The red curve also includes $1 / f$, or flicker noise. In this case, Eq 2 cannot be analytically solved and the linewidth must be numerically calculated. Lastly, the green-dashed curve includes the carrier induced frequency noise as well. It is noteworthy how the last effect has no influence on either 3 or 20 $\mathrm{dB}$ linwewidth. To further investigate this, we performed several sweeps and observed the impact of lorentzian on the 3 and $20 \mathrm{~dB}$ linewidth. Table 1 shows the parameters of the model as well as the ranges of each sweep. 

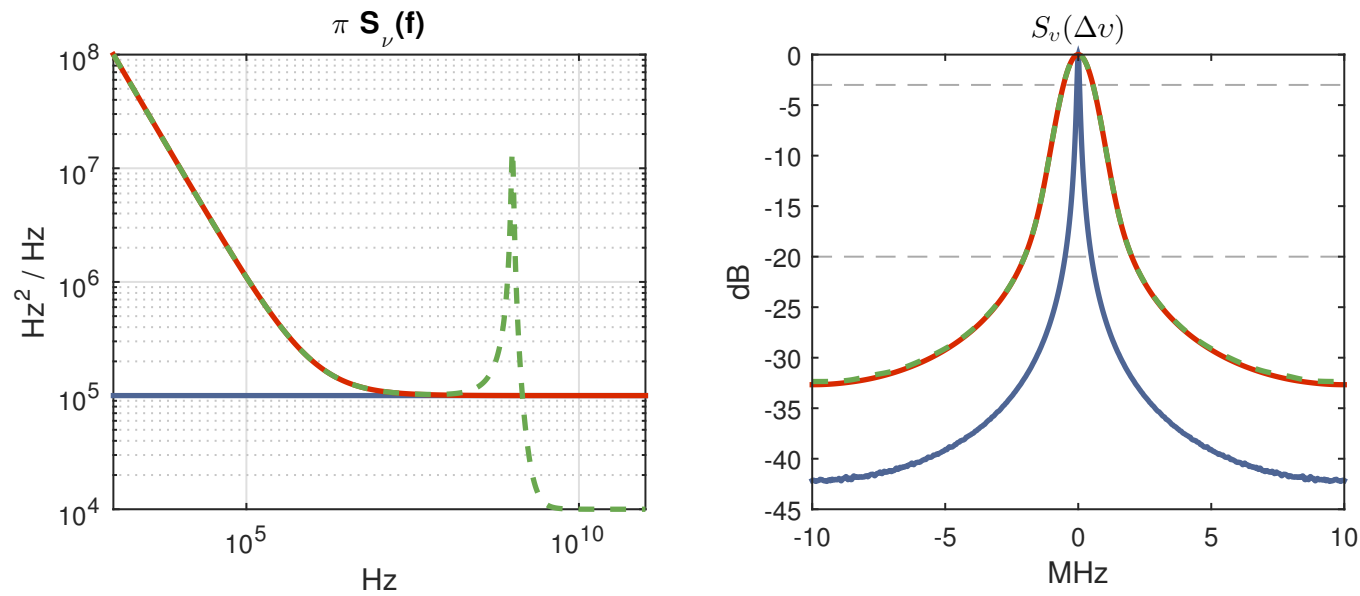

Figure 2: Frequency noise PSD (left) and optical spectrum (right) for only white frequency noise (blue), including flicker noise (red), and including carrier induced frequency noise (green)

Each parameter is swept while keeping the rest of the parameters fixed to their default value. The results are presented in figure 3. We can observe a linear relation with respect the $S_{v}(\Delta v)$ (figure 3a). This is due to the fact that this parameter scales the amount of white frequency noise evenly throughout the FM noise spectrum. In case of $\Delta_{v(1 / f)}$ (figure $3 \mathrm{~b}$ ), the 3 and $20 \mathrm{~dB}$ linewidths take the shape of $\sqrt{\Delta_{v(1 / f)}}$. For $f_{R}$ parameter, we observe a $0.15 \mathrm{~dB}$ deviation at low resonance frequencies for the $20 \mathrm{~dB}$ linewidth, and no deviation above $2 \mathrm{GHz}$. As for $K$ and $\alpha$ parameters (figures 3d and e), there is no impact on either 3 or $20 \mathrm{~dB}$ linewidth for the values under study.

\begin{tabular}{llll} 
Parameter & Default & Range & Units \\
\hline$\Delta_{v(1 / f)}$ & 100 & $100-900$ & $\mathrm{kHz}$ \\
$\Delta_{\text {vint }}$ & $10^{4}$ & $1-10^{4}$ & $\mathrm{~Hz}$ \\
$f_{R}$ & 10 & $1-10$ & $\mathrm{GHz}$ \\
$K$ & 0.15 & $0.1-1$ & $\mathrm{~ns}$ \\
$\alpha$ & 3 & $0.1-10$ & -
\end{tabular}

Table 1: Parameters for linewidth sweeps

\section{IMPACT ON SYSTEM PERFORMANCE}

The effect of each parameter should be study in terms of system performance. Intrinsic linewidth is studied in most of the studies reviewed in section 2. The relation depends on the specific CPR technique but tends to be linear. ${ }^{7} 1 / f$, or flicker noise has also been extensively studied, ${ }^{22-24}$ where the penalty seems to increase exponentially with the amount of $1 / \mathrm{f}$ noise. For carrier induced frequency noise, however, no studies has been

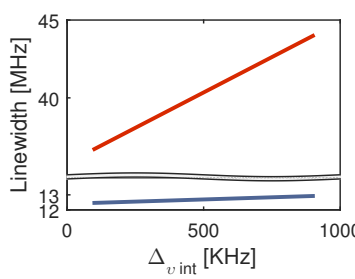

(a)

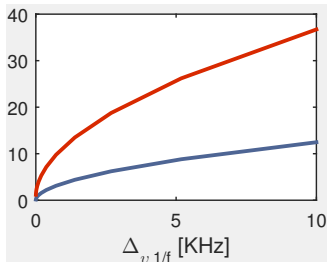

(b)

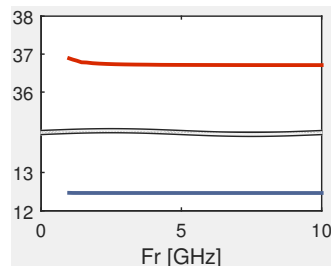

(c)

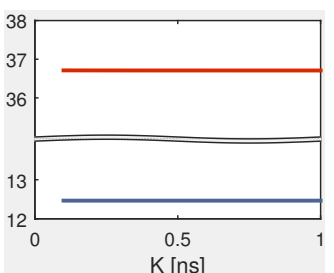

(d)

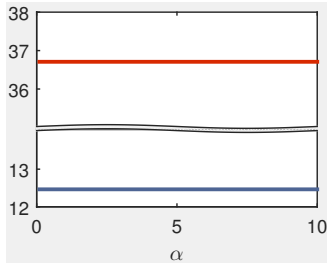

(e)

Figure 3: Impact of model parameters on 3 (blue) and 20 (red) dB linewidth for each parameter of the model (eq.1). 


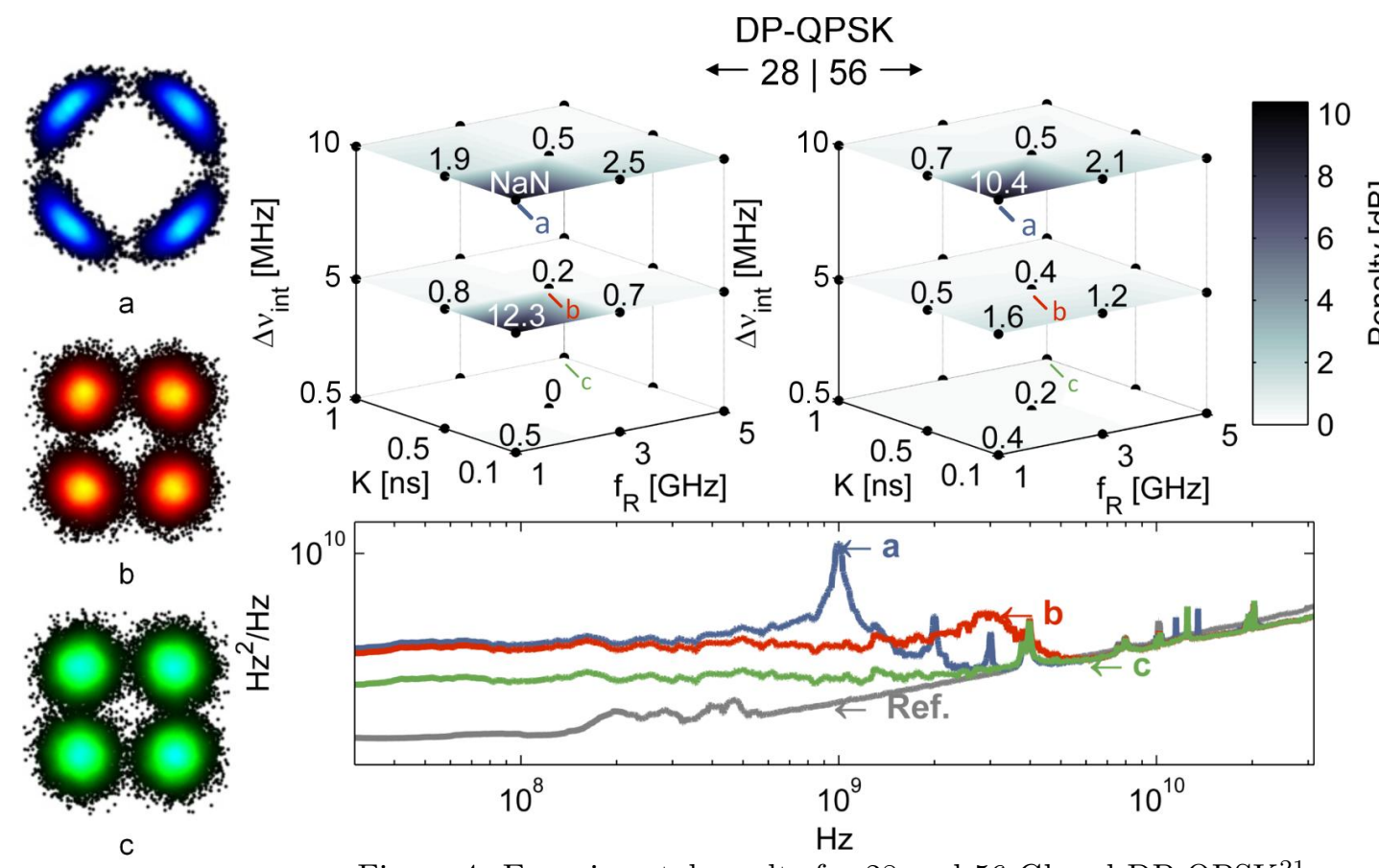

Figure 4: Experimental results for 28 and 56 Gbaud DP-QPSK ${ }^{21}$

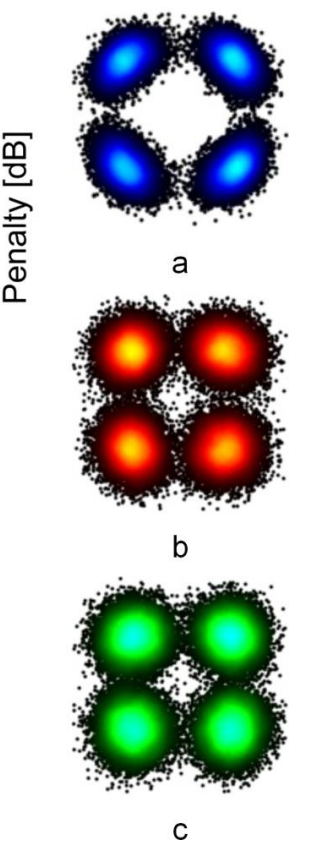

found on system performance. This noise is mainly governed by the resonance frequency and the K-factor and is the main topic of our research. In our previous experiments, the impact of semiconductor laser frequency noise was systematically studied on 28 and 56 Gbaud DP-QPSK systems with for up to $500 \mathrm{~km}$ standard single mode fiber (SSMF). The experimental setup is detailed $i^{21}$ and consisted of a standard DP-QPSK transmitter and receiver, with $100 \mathrm{kHz}$ external cavity lasers used for both the local oscillator and the transmitter. To emulate specific phase noise profiles, we used a LiNbO3 phase modulator and an arbitrary waveform generator at $50 \mathrm{GS} / \mathrm{s}$ to control the phase noise spectrum of the transmitter laser. For data demodulation, we use the a standard DSP chain based on. ${ }^{25}$ The CPR algorithm used was a standard DDPLL based on. ${ }^{10}$

Figure 4 summarizes the results in back to back (B2B) configuration. The 3D plots show the penalty with respect to OSNR at the receiver sensitivity under no added phase noise, for different phase noise conditions, where the x-axis represents $f_{R}$ for 1,3 and $5 \mathrm{GHz}$. The y-axis represents the $K$-factor for $0.1,0.5$ and 1 ns. And the z-axis represents $\Delta_{\text {vint }}$ for $0.5,5$ and $10 \mathrm{MHz}$. The OSNR penalty is color coded, where a larger intensity represents larger penalty. We define the receiver sensitivity at a BER of $2 \cdot 10^{-3}$. The OSNR values at this baudrate under no added phase noise were 11.3 and $14.5 \mathrm{~dB}$ for 28 and 56 Gbaud respectively; both B2B and after $500 \mathrm{~km}$ SSMF. A total of 27 BER curves were measured from where the OSNR at receiver sensitivity was obtained. This was measured for both 28 Gbaud (left) and 56 Gbaud (right) DP-QPSK, both B2B and after $500 \mathrm{~km}$ SSMF with no observable penalty due to transmission. Three cases were selected covering all values for the parameters $\left[f_{R}, K, \Delta_{v i n t}\right]$, under study, from the worst case to the best. The cases are defined as a $=$ $[1,0.1,10], \mathrm{b}=[3,0.5,5]$ and $\mathrm{c}=[5,1,0.5]$ for both 28 and 56 Gbaud signals. The frequency noise power spectral density $S_{v}(f)$ of each case is also presented in Fig 4 . The position of the labels within the plot also indicates the position of each resonance frequency. A reference spectrum without any added phase noise is also included. In addition, the received constellations for the x-polarization at each case are presented. Note that, for the 28 Gbaud DP-QPSK signal, the obtained BER in case a) was below the sensitivity, and therefore the OSNR is represented as not a number $(\mathrm{NaN})$. On the other hand, the penalty observed between the reference phase noise and case c) was negligible.

The results show that the influence of the resonance peak has a major impact on system performance. As expected, the penalty is reduced as the boudrate is increased. It is interesting to observe that up to $10 \mathrm{MHz}$ of intrinsic linewidth can be tolerated within $0.5 \mathrm{~dB}$ penalty as long as the resonance frequency and the K-factor are above $3 \mathrm{GHz}$ and $0.5 \mathrm{~ns}$ respectively. 


\section{CONCLUSIONS}

This paper addresses the status of CPR algorithms for semiconductor phase noise tolerant coherent transmission systems. A state-of-the-art review for CPR techniques is performed, concluding that most of the techniques are benchmarked agains laser linewidth, which inherently assumes lorentzian lineshape. We then show how carrier induced frequency noise does not contribute to the $3 \mathrm{~dB}$ laser linewidth. However, it is identified that this type of noise plays an important factor on system performance. The results lead to the conclusion that $3 \mathrm{~dB}$ linewidth is not a good estimator of system performance for semiconductor laser frequency noise. Future lines of work include exploring the impact on 16-DPQAM systems and including the dynamics of carrier induced frequency noise on CPR algorithms. We identify Kalman CPR as one of the potential candidates to include this effect, although a feedforward strucutre would be prefered to reduce the DSP complexity.

\section{ACKNOWLEDGMENTS}

Projects GRIFFON, gr. \#324391, ICONE, gr. \#608099, and the Villum Foundation Young Investigator program, and the Swedish Institute (SI) are acknowledged. The equipment was funded by Knut and Alice Wallenberg foundation.

\section{REFERENCES}

[1] Han, Y. and Li, G., "Coherent optical communication using polarization multiple-input-multiple-output," Opt. Express 13(19), 7527 (2005).

[2] Kikuchi, K., "Characterization of semiconductor-laser phase noise and estimation of bit-error rate performance with low-speed offline digital coherent receivers.," Opt. Express 20, 5291-302 (Feb. 2012).

[3] Gallion, P. and Debarge, G., "Quantum phase noise and field correlation in single frequency semiconductor laser systems," IEEE J. Quantum Electron. 20, 343-349 (Apr. 1984).

[4] Chraplyvy, A., Liou, K., Tkach, R., Eisenstein, G., Jhee, Y., Koch, T., Anthony, P., and Chakrabarti, U., "Simple narrow-linewidth $1.5 \mu \mathrm{m}$ InGaAsP DFB external-cavity laser," Electron. Lett. 22(2), 88 (1986).

[5] Kikuchi, K. and Okoshi, T., "Measurement of FM Noise, AM Noise, and Field Spectra of 1. 3 p m InGaAsP FB Lasers and Determination of the Linewidth Enhancement Factor," IEEE J. Quantum Electron. QE21(11), 1814-1818 (1985).

[6] Di Domenico, G., Schilt, S., and Thomann, P., "Simple approach to the relation between laser frequency noise and laser line shape.," Appl. Opt. 49, 4801-7 (Sept. 2010).

[7] Taylor, M. G., "Phase Estimation Methods for Optical Coherent Detection Using Digital Signal Processing," J. Light. Technol. 27, 901-914 (Apr. 2009).

[8] Pfau, T., Hoffmann, S., and Noe, R., "Hardware-Efficient Coherent Digital Receiver Concept With Feedforward Carrier Recovery for M-QAM Constellations," J. Light. Technol. 27, 989-999 (Apr. 2009).

[9] Gao, Y., Lau, A. P. T., and Lu, C., "Modulation-Format-Independent Carrier Phase Estimation for Square M-QAM Systems," IEEE Photonics Technol. Lett. 25, 1073-1076 (June 2013).

[10] Zibar, D., Peucheret, C., Jeppesen, P., and Monroy, I., "Digital Coherent Receiver for Phase-Modulated Radio-Over-Fiber Optical Links," IEEE Photonics Technol. Lett. 21, 155-157 (Feb. 2009).

[11] Gianni, P., Corral-Briones, G., Rodriguez, C., and Hueda, M. R., "Compensation of Laser Frequency Fluctuations and Phase Noise in 16-QAM Coherent Receivers," IEEE Photonics Technol. Lett. 25, 442-445 (Mar. 2013).

[12] Zhang, S., Kam, P. Y., Chen, J., and Yu, C., "Decision-aided maximum likelihood detection in coherent optical phase-shift-keying system," Opt. Express 17, 703 (Jan. 2009).

[13] Huang, D. and Cheng, T.-h., "Decision-aided carrier phase estimation with selective averaging for low-cost optical coherent communication," 2013 9th Int. Conf. Information, Commun. Signal Process. , 1-3 (Dec. 2013). 
[14] Zibar, D., Carvalho, L., Piels, M., Doberstein, A., Diniz, J., Nebendahl, B., Franciscangelis, C., Estaran, J., Haisch, H., Gonzalez, N. G., de Oliveira, J. R. F., and Monroy, I. T., "Bayesian filtering for phase noise characterization and carrier synchronization of up to $192 \mathrm{~Gb} / \mathrm{s}$ PDM 64-QAM," in [Opt. Commun. (ECOC), 2014 Eur. Conf.], 1-3 (Sept. 2014).

[15] Noé, R., Hoffmann, S., Wördehoff, C., Al-Bermani, A., and El-Darawy, M., "Advances in Coherent Optical Modems and 16-QAM Transmission with Feedforward Carrier Recovery," in [Components], Li, G. and Jäger, D. S., eds., 7960, 79600L-79600L-9 (Jan. 2011).

[16] Viterbi, A. and Viterbi, A., "Nonlinear estimation of PSK-modulated carrier phase with application to burst digital transmission," IEEE Trans. Inf. Theory 29, 543-551 (July 1983).

[17] Fatadin, I., Ives, D., and Savory, S. J., "Laser Linewidth Tolerance for 16-QAM Coherent Optical Systems Using QPSK Partitioning," IEEE Photonics Technol. Lett. 22, 631-633 (May 2010).

[18] Ip, E. and Kahn, J. M., "Feedforward carrier recovery for coherent optical communications," J. Light. Technol. 25, 2675-2692 (2007).

[19] Li, X., Cao, Y., Yu, S., Gu, W., and Ji, Y., "A Simplified Feedforward Carrier Recovery Algorithm for Coherent Optical QAM System," J. Light. Technol. 29, 801-807 (Mar. 2011).

[20] Zhou, X., Zhong, K., Gao, Y., Lu, C., Lau, A. P. T., and Long, K., "Modulation-format-independent blind phase search algorithm for coherent optical square M-QAM systems.," Opt. Express 22, 24044-54 (Oct. 2014).

[21] Iglesias Olmedo, M., Pang, X., Udalcovs, A., Schatz, R., Zibar, D., Jacobsen, G., Popov, S., and Tafur Monroy, I., "Impact of Carrier Induced Frequency Noise from the Transmitter Laser on 28 and 56 Gbaud DP-QPSK Metro Links," in [Asia Commun. Photonics Conf.], ATh1E.1 (2014).

[22] Shi, K., Smyth, F., Anandarajah, P. M., Reid, D., Yu, Y., and Barry, L. P., "Linewidth of SG-DBR laser and its effect on DPSK transmission," Opt. Commun. 283, 5040-5045 (Dec. 2010).

[23] Huynh, T. N., Nguyen, L., and Barry, L. P., "Phase Noise Characterization of SGDBR Lasers Using Phase Modulation Detection Method With Delayed Self-Heterodyne Measurements," J. Light. Technol. 31, 13001308 (Apr. 2013).

[24] Matsuda, K., Bessho, H., Hasegawa, K., Yoshida, T., and Ishida, K., "A Study of Laser White and Brownian FM Noise in Coherent QPSK Signals," Opt. Fiber Commun. Conf., W4K.4 (2014).

[25] Borkowski, R., Zibar, D., and Tafur Monroy, I., "Anatomy of a Digital Coherent Receiver," IEICE Trans. Commun. E97.B(8), 1528-1536 (2014). 